\title{
Correlating Antiagglomerant Performance with Gas Hydrate Cohesion
}

\author{
Anh Phan, ${ }^{1}$ Michail Stamatakis, ${ }^{1}$ Carolyn A. $\mathrm{Koh}^{2}$ and Alberto Striolo ${ }^{1, *}$ \\ ${ }^{1}$ Department of Chemical Engineering, University College London, London WC1E 7JE, UK \\ ${ }^{2}$ Center for Hydrate Research, Chemical \& Biological Engineering Department, Colorado School of \\ Mines, Golden, Colorado 80401, United States
}

Supplementary Information

*Corresponding Author

a.striolo@ucl.ac.uk 

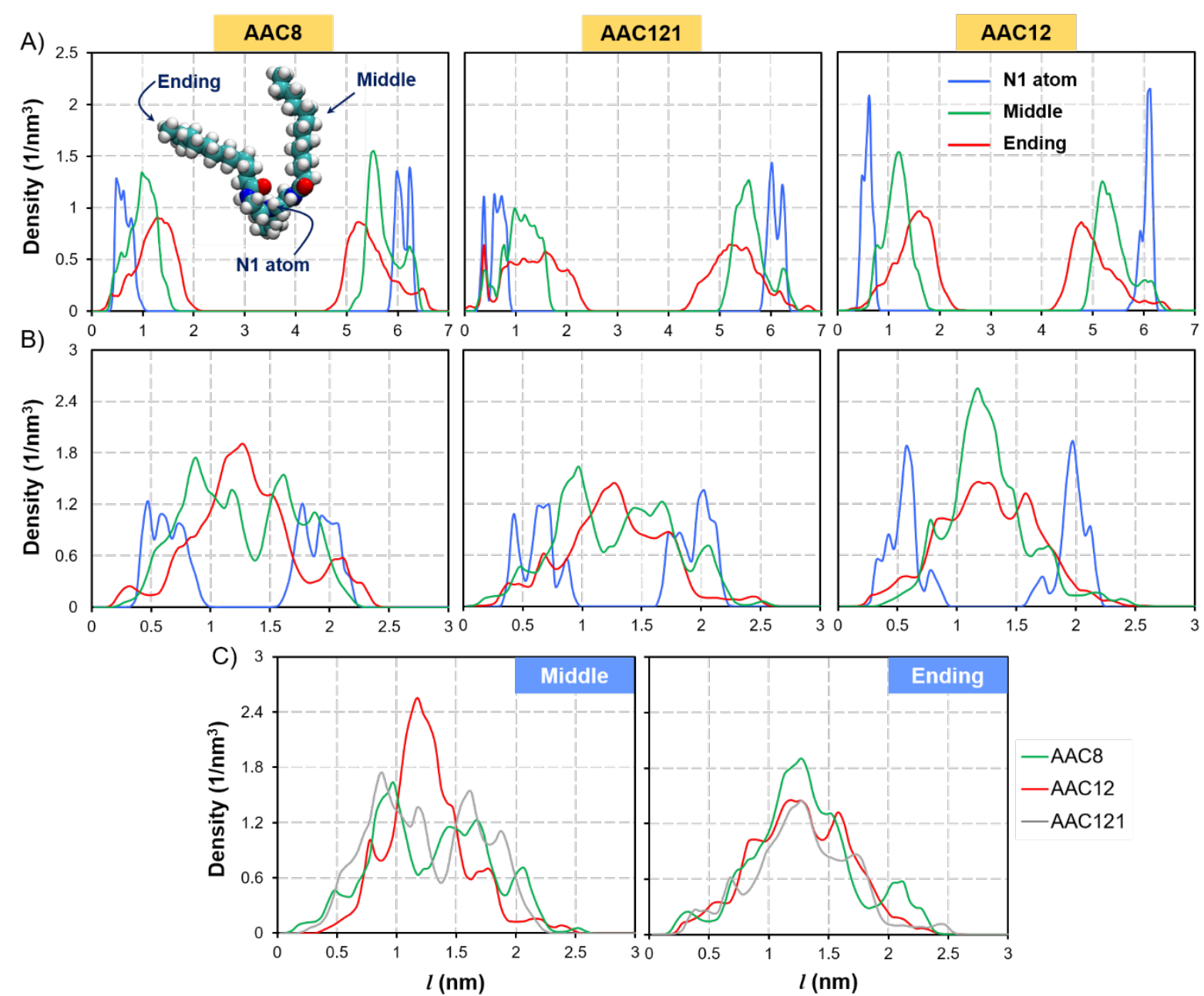

Figure S1. Density profiles of AA layers confined between two hydrate particles when they are far from each other (panel A) and when they are brought at distance $l=2.51 \mathrm{~nm}$ (panel B), where the repulsive barrier is located in the PMF profile obtained for systems with AAC12, as shown in Figure 2 of the main text. Results are shown for $\mathrm{N}^{+}$atom (blue), middle (green) and ending (red) carbon atoms of AAC8 (left), AAC121 (middle), and AAC12 (right) tails along the Z-direction (perpendicular to the hydrate surfaces). C) Density profiles of middle (left) and ending (right) carbon atoms of AAC8 (green), AAC121 (grey), and AAC12 (red) tails along the Z-direction when the two hydrate particles are brought close near one another at distance $l=2.51 \mathrm{~nm}$

In Figure S1, we report the results of density profiles of AA layers confined between the two hydrate particles when they are far from each other (panel A) and when they are at distance $l=2.51 \mathrm{~nm}$ (panel B). Results are shown for $\mathrm{N}^{+}$atom (see Figure 1B in the main text) (blue), middle (green) and end (red) carbon atoms of AAC8 (left), AAC121 (middle), and AAC12 (right) tails along the Z-direction (perpendicular to the hydrate surfaces). We do not observe significant differences in the density profiles for different AAs, e.g., AAC8, AAC121, and AAC12 when the two hydrate particles are far from each other (panel A). However, when the two hydrate particles are at $l=2.51 \mathrm{~nm}$, the density peak obtained for the system with AAC12 is noticeably more pronounced and narrower compared to those found for 
systems with AAC8 and AAC121 (left, panel C). Note that the total number of middle carbon atoms of tails of AAC8, AAC121 and AAC12 are the same. This suggests that AAC12 might not be as flexible as the other AAs considered, i.e., AAC8 and AAC121; the presence of pronounced density peak for the middle carbon atoms of AAC12 tails when the two hydrate particles are near each other could be related to the free energy barrier in the PMF results. We do not observe significant differences in the results for density profiles obtained for the ending carbon atoms of tails of AAC8 (green), AAC121 (grey) and AAC12 (red) when the two hydrate particles are brought near one another.

In Table S1, we report data extracted from the PMF profiles, shown in Figure 2 of the main text.

Table S1. Locations of minimum and local maxima in the PMF profiles and locations where attraction initiates.

\begin{tabular}{lcccccc} 
& \multicolumn{2}{c}{ Minimum $(\AA)$} & \multicolumn{3}{c}{ Local Maximum $(\AA)$} & \multicolumn{2}{c}{ Coalescence Initiation $(\AA)$} \\
\cline { 2 - 7 } & C12 & C7 & C12 & C7 & C12 & C7 \\
\hline AAC8-0.44 & 12.5 & 13.6 & & & 26.9 & 26.9 \\
AAC12-0.44 & 13.5 & 11.1 & 21.6 & 23.7 & 17.2 & 20.6 \\
AAC121-0.44 & 13.3 & 11.5 & 25.5 & 26.5 & 24.4 & 24.9 \\
AAC171-0.28 & 10.5 & 11.5 & 23.5 & 25.4 & 20.0 & 24.5 \\
AAC171-0.44 & 10.8 & 13.6 & 20.2 & 21.6 & 14.4 & 17.4
\end{tabular}

The force-distance profiles shown in Figure 3 of the main text are reminiscent of a Lennard-Jones force (with parameters $\varepsilon$ and $\sigma$ ) superimposed to a repulsive peak (parameters $r_{o}, b$, and $n$ ), yielding the following expression: ${ }^{1}$

$$
F=4 \varepsilon\left(\frac{12 \sigma^{12}}{r^{13}}-\frac{6 \sigma^{6}}{r^{7}}\right) \times\left(1-\sqrt{\pi} \exp \left[\frac{-\left(r-r_{o}\right)^{2}}{b}\right] \times n\right)
$$

The procedure of fitting simulated force data points while removing outliers follows three steps:

1. Fit simulated force data points to Eq. (1) via adjusting the parameters $\varepsilon, \sigma, r_{o}, b$, and $n$ employing a non-linear regression with the Levenberg-Marquardt algorithm.

2. Examine the residuals of the fit and identify whether one or more values are outliers. To do this, we applied the method of outliner identification developed by Motulsky and Brown ${ }^{2}$ based on the False 
Discovery Rate (FDR) approach of testing for multiple comparisons. The details about the numerical procedure to detect outliers follows these steps are described by Motulsky and Brown. ${ }^{2}$

3. Remove the outliers and implement non-linear regression on the remaining data.

4. Employ the chi-squared test and degrees of freedom for fitting parameters to perform a 'goodness of fit' test. An iterative procedure that minimizes the reduced chi-square value to obtain the optimal parameter values is implemented during the nonlinear curve fitting performance. The reduced chisquare was calculated by dividing the residual sum of squares by the degrees of freedom.

5. Complete the fitting with a minimal value of reduced chi-square and a high value of the coefficient of determination $\left(R^{2}\right)$ for the model parameters.

In Table S2, we report the resultant fitted parameters.

Table S2. Values of the fitting parameters of the force - distance curve model described in Eq. (1) for all systems considered

\begin{tabular}{lccccc} 
& $\varepsilon\left(\mathrm{kcal}^{\mathrm{mol}}{ }^{-1} \cdot \mathrm{nm}^{-1}\right)$ & $\sigma(\mathrm{nm})$ & $\mathrm{r}_{\mathrm{o}}(\mathrm{nm})$ & $\mathrm{b}\left(\mathrm{nm}^{2}\right)$ & $\mathrm{n}$ \\
\hline AAC8 $-\mathrm{C} 12$ & $0.115 \pm 0.005$ & $1.108 \pm 0.009$ & $1.950 \pm 0.016$ & $0.190 \pm 0.002$ & $-1.45 \mathrm{E} 6 \pm 0.07 \mathrm{E} 6$ \\
AAC12 - C12 & $0.008 \pm 0.002$ & $1.934 \pm 0.024$ & $2.500 \pm 0.031$ & $0.150 \pm 0.002$ & $-1.15 \mathrm{E} 3 \pm 0.31 \mathrm{E} 3$ \\
AAC121-C12 & $0.015 \pm 0.002$ & $1.190 \pm 0.003$ & $1.950 \pm 0.005$ & $0.190 \pm 0.001$ & $-1.84 \mathrm{E} 6 \pm 0.27 \mathrm{E} 6$ \\
AAC171-C12 & $1.137 \pm 0.096$ & $0.854 \pm 0.002$ & $1.950 \pm 0.005$ & $0.230 \pm 0.001$ & $-1.09 \mathrm{E} 1 \pm 0.09 \mathrm{E} 1$ \\
AAC8 - C7 & $0.324 \pm 0.064$ & $1.212 \pm 0.005$ & $1.950 \pm 0.008$ & $0.190 \pm 0.001$ & $-1.10 \mathrm{E} 2 \pm 0.22 \mathrm{E} 2$ \\
AAC12 - C7 & $0.466 \pm 0.100$ & $1.005 \pm 0.008$ & $1.950 \pm 0.016$ & $0.190 \pm 0.002$ & $-2.07 \mathrm{E} 2 \pm 0.45 \mathrm{E} 2$ \\
AAC121-C7 & $0.098 \pm 0.011$ & $1.032 \pm 0.006$ & $1.950 \pm 0.011$ & $0.190 \pm 0.001$ & $-1.28 \mathrm{E} 3 \pm 0.14 \mathrm{E} 3$ \\
AAC171 - C7 & $0.046 \pm 0.003$ & $1.133 \pm 0.004$ & $1.950 \pm 0.007$ & $0.190 \pm 0.001$ & $-1.39 \mathrm{E} 3 \pm 0.10 \mathrm{E} 3$ \\
\hline
\end{tabular}

\section{References}

1. Vo, M. D.; Papavassiliou, D. V. Interaction between Polymer-Coated Carbon Nanotubes with Coarse-Grained Computations. Chem Phys Lett 2017, 685, 77-83.

2. Motulsky, H. J.; Brown, R. E. Detecting Outliers When Fitting Data with Nonlinear Regression - a New Method Based on Robust Nonlinear Regression and the False Discovery Rate. Bmc Bioinformatics 2006, 7. 